\title{
Cost-effectiveness analysis of traditional Chinese medicine combined with antiviral drugs for hepatitis B cirrhosis based on Markov model
}

\section{Zhiheng Wang}

Beijing University of Chinese Medicine https://orcid.org/0000-0002-1123-2777

\section{Mengpei Zhang}

Beijing University of Chinese Medicine

Haoxiang Zhang

Beijing University of Chinese Medicine

Kaini Zuo

Beijing University of Chinese Medicine

Jie Pan

Beijing University of Chinese Medicine

Xiaomei Wang

Beijing University of Chinese Medicine

Zhu Wentao ( $\nabla$ wentao67@126.com )

\section{Research}

Keywords: Chinese medicine, antiviral drugs, Hepatitis B cirrhosis, cost-effectiveness analysis, Markov model

Posted Date: May 15th, 2020

DOl: https://doi.org/10.21203/rs.3.rs-26998/v1

License: (c) (i) This work is licensed under a Creative Commons Attribution 4.0 International License. Read Full License 


\section{Abstract}

Background Hepatitis B cirrhosis brings a heavy burden to the health care system all over the world, and traditional Chinese medicine had a good effect in the treatment of hepatitis B cirrhosis. To correctly understand the clinical effect of Chinese medicine combined with antiviral drugs in the treatment of hepatitis B cirrhosis, the long-term therapeutic effect of traditional Chinese medicine combined with antiviral drugs in the treatment of hepatitis B cirrhosis was simulated using Markov model analysis.

Methods We used four-state Markov modelling with a probabilistic cohort analysis to calculate the cost per quality-adjusted life year (QALY) gained. A one-year cycle length was applied. The best available evidence provided the model with data on the state of hepatitis B cirrhosis, risk of mortality, utility weight, and probability of transition between disease costs. A 3\% discount rate was used for costs and QALYs. Cost-effectiveness acceptability curves were presented to assist decision makers.

Results The model indicated that the combination of traditional Chinese medicine and antiviral therapy was cost-effective, and the result showed that there was some uncertainty. In the treatment of hepatitis B cirrhosis simulated by Markov model for 20 years, the combination of traditional Chinese medicine and anti-virus drugs increased 1.48 QALYs per patient compared with the use of antiviral drugs alone, and the total cost saved was $45,551.61$ yuan. The total cost saving was mainly due to the reduction of number of hospitalizations caused by serious complications.

Conclusion The combination of traditional Chinese medicine and antiviral in the treatment of hepatitis B cirrhosis could save the cost of hospitalization and improve the health output, which was a better outcome evaluated by pharmacoeconomics.

\section{Background}

According to statistics published by the World Health Organization (WHO), there were about 257 million patients infected with chronic hepatitis B virus (HBV) in the world, and $68 \%$ of them were in Africa and the Western Pacific, were China contributed a significant proportion in the total number [1]. In 2018, the incidence rate of viral hepatitis in China was $92.15 / 100,000$, with a mortality rate of $0.04 / 100,000$. Among patients with cirrhosis and hepatocellular carcinoma in China, $77 \%$ and $84 \%$ were caused by HBV respectively [4]. In the diagnosis of liver cirrhosis, cirrhosis was often divided into compensatory period and decompensated period according to the occurrence of severe complications such as ascites, esophagogastric varices bleeding, and hepatic encephalopathy. Hepatitis B cirrhosis and related diseases could impose a heavy economic burden on individuals and families.

The main treatment of hepatitis B cirrhosis was antiviral drugs, combined with the actual situation of patients and liver protection drugs. As nucleoside analog, Entecavir could effectively inhibit the replication of hepatitis $B$ virus and delay the deterioration of the disease. It was one of the first choice of hepatitis B antiviral drugs recommended by the Guidelines for the Prevention and Treatment of Chronic Hepatitis B (2019 edition) [4]. With the continuous exploration of the value of traditional Chinese 
medicine in clinical medicine, more traditional Chinese medicine such as Ganshuang Granules and Compound Biejia Ruangan Tablets were used in the clinical treatment of hepatitis B cirrhosis and had good therapeutic effects. In this study, a Markov model was established to simulate the long-term treatment impact of traditional Chinese medicine combined with antiviral drugs versus antiviral drugs alone in the treatment of hepatitis B cirrhosis, and evaluate the cost-effectiveness of such treatments.

\section{Materials And Methods}

\section{Analysis Method}

Studies showed that the median survival time of hepatitis B cirrhosis was $10 \sim 12$ years [16], and some patients died about 25 months after treatment [26]. Therefore, this study assumed that the average survival time of patients with hepatitis $B$ cirrhosis was 11 years, and the minimum survival time was 2 years. According to the formula: maximum value $\approx$ average value* 2 - minimum value, the maximum survival period of patients with hepatitis B cirrhosis was estimated to be 20 years, Therefore, the simulation period of Markov model in this paper was set as 20 years. In this study, a Markov model with 1-year simulation cycle was constructed to simulate the health status of patients with hepatitis $B$ cirrhosis treated with traditional Chinese medicine combined with antiviral drugs and antiviral drugs alone for 20 years, and a cost-utility analysis was conducted. The cost and health output were discounted at a discount rate of $3 \%$ based on the price level in 2018 . The stability of the model was evaluated by univariate sensitivity analysis and probabilistic sensitivity analysis.

\section{Data Sources}

The model parameters mainly included the probability of clinical disease outcomes, direct medical costs, direct non-medical costs and health utility values, etc. The data were retrieved from relevant research literatures of hepatitis B, and drug price was retrieved from the website China 315 drug prices.

\section{Model Analysis}

\section{Basic Assumptions of Markov Model}

(1) The clinical treatment of cirrhosis mainly depended on the symptomatic treatment and the improvement of liver function, and there were few curative treatment to reverse the course [21]. Therefore, the state of compensatory cirrhosis was set as the initial disease state of the Markov model in this study.

(2) The probability of cirrhosis re-compensation was small, and there was no accurate definition and unified diagnostic criteria for re-compensation cirrhosis [4]. The development of diseases from recompensated of cirrhosis to compensated was not considered. and once liver cancer occured, it was irreversible. Therefore, this study assumed that the probability of liver cancer to compensate cirrhosis and re-compensated cirrhosis were 0. 
(3) The incidence rate of chronic diseases after liver transplantation such as metabolic diseases, chronic kidney disease and cardiovascular diseases was high, and the incidence of chronic diseases after liver was increasing year by year [22], the burden of disease after liver transplantation was higher. At present, there were not many cases of liver transplantation in China. Therefore, the disease status of liver transplantation was not considered.

(4) As shown in Table 1, since there was no large-scale cohort study data on the combination of traditional Chinese medicine and antiviral drugs in the treatment of hepatitis B cirrhosis, the relative risk $(\mathrm{RR})$ and mean difference (MD) in the meta-analysis result of the systematic evaluation of Chinese medicine for the treatment of chronic hepatitis B virus carriers, liver fibrosis and cirrhosis by Feng Shuo [11] were used to determine the treatment parameters of Chinese medicine combined with antiviral drugs. The research suggested that: $\otimes$ Taking all-cause mortality as the evaluation index, Chinese medicine combined with antiviral drugs was better than antiviral drugs alone, and its $\mathrm{RR}=0.53$; $\triangle \mathrm{Based}$ on the incidence rate of liver cancer, there was no significant difference between the combination of traditional Chinese medicine and antiviral drugs and the use of antiviral drugs alone; $₫$ Taking the quality of life as an evaluation index, Chinese medicine combined with antiviral drugs was better than antiviral drugs alone, which could improve the score of quality of life and has significant difference, its $M D=10.11$; $\triangle A c c o r d i n g$ to the evaluation index of complication rate, the combination of traditional Chinese medicine and antiviral drugs was superior to the comprehensive treatment of Western medicine, and its RR $=0.56$.

Table 1

Parameters and data sources of the influence of traditional Chinese medicine combined with antiviral drugs in the Markov model

\begin{tabular}{|c|c|c|c|c|c|}
\hline $\begin{array}{l}\text { Influencing parameters of } \\
\text { Chinese medicine combined } \\
\text { with antiviral drugs }\end{array}$ & $\begin{array}{l}\text { Model } \\
\text { parameters }\end{array}$ & $\begin{array}{l}\text { Sample } \\
\text { size }\end{array}$ & Value & Range & Distributed \\
\hline Complication rate ${ }^{[11]}$ & rr_dc & 169 & 0.56 & $(0.35,0.91)$ & $\begin{array}{l}\text { Log- } \\
\text { normal }\end{array}$ \\
\hline All-cause mortality ${ }^{[11]}$ & rr_die & 238 & 0.53 & $(0.16,0.99)$ & $\begin{array}{l}\text { Log- } \\
\text { normal }\end{array}$ \\
\hline Quality of life ${ }^{[11]}$ & rr_u & 234 & 0.1011 & $(0.0681,0.1341)$ & Normal \\
\hline Hospitalization [11] & rr_t & 169 & 0.56 & $(0.5,1)$ & Triangular \\
\hline \multicolumn{6}{|c|}{$\begin{array}{l}\text { Note: rr_dc: Compared with using antiviral drugs alone, influence coefficient of probability of Chinese } \\
\text { medicine combined with antiviral drugs on the transition from compensated cirrhosis to } \\
\text { decompensated cirrhosis; rr_die: Compared with using antiviral drugs alone, the probabilistic influence } \\
\text { coefficient of combined antiviral drugs with traditional Chinese medicine on the outcome of } \\
\text { compensated cirrhosis and decompensated cirrhosis to death; } \boldsymbol{I}_{-} \mathrm{u} \text { : The proportion of life quality } \\
\text { improvement of patients with compensated cirrhosis and decompensated cirrhosis treated with } \\
\text { Chinese medicine combined with antiviral drugs compared with those treated with antiviral drugs } \\
\text { alone; rr_t: Compared with using antiviral drugs alone, the influence coefficient of combination of } \\
\text { traditional Chinese medicine and antiviral drugs on hospitalization times of patients, when rr_t }=1 \text {, it } \\
\text { meant that the number of hospitalizations for Chinese medicine combined with antiviral drugs and } \\
\text { antiviral drugs alone was the same. }\end{array}$} \\
\hline
\end{tabular}




\section{Model Parameter Setting}

(1) Probability of state transition: As shown in Table 2, the probability of transition between various disease states of hepatitis B cirrhosis was adopted by Giovanna Fattovich et al. [6], and Xu Biao et al. [7]. The research used a retrospective cohort study to research the outcome and development of chronic hepatitis B disease.

Table 2

Probability parameters and data sources of disease state transition in Markov model

\begin{tabular}{|c|c|c|c|c|}
\hline \multicolumn{2}{|c|}{ Transfer probability (years) } & \multirow[t]{2}{*}{ Value } & \multirow[t]{2}{*}{ Range } & \multirow[t]{2}{*}{ Distributed } \\
\hline Initial state & Final state & & & \\
\hline \multicolumn{5}{|l|}{$C C$} \\
\hline & $D C^{[6],[14]}$ & 0.07 & $(0.03,0.1)$ & Beta \\
\hline & $H C C^{[6]}$ & 0.034 & $(0.01,0.13)$ & Beta \\
\hline & $D i e^{[7]}$ & 0.0074 & & Beta \\
\hline \multicolumn{5}{|l|}{$D C$} \\
\hline & $H C C^{[6],[17],[18]}$ & 0.034 & $(0.01,0.1)$ & Beta \\
\hline & $D i e^{[15],[17]}$ & 0.144 & $(0.058,0.30)$ & Beta \\
\hline \multicolumn{5}{|l|}{$\mathrm{HCC}$} \\
\hline & $D i e^{[15],[17]}$ & 0.4 & $(0.32,0.56)$ & Beta \\
\hline
\end{tabular}

(2) Health output: As shown in Table 3, the health output index QALYs used Wong [8] to obtain the utility value of each state of hepatitis $B$ cirrhosis according to the standard game method and time trade-off method. The utility value obtained by Wong et al. [8] was consistent with the utility value of each disease state of hepatitis B cirrhosis confirmed by Chen Dong et al. [9], David L and Veenstra et al. [10] in two rounds of expert consultation in China. 
Table 3

Health utility parameters and data sources in Markov model

\begin{tabular}{|lll|}
\hline Health utility value & Value & Distributed \\
\hline$C C^{[8-10]}$ & 0.57 & Beta \\
\hline$D C^{[8-10]}$ & 0.26 & Beta \\
\hline$H C C^{[8-10]}$ & 0.31 & Beta \\
\hline$D i{ }^{[8-10]}$ & 0 & \\
\hline
\end{tabular}

(3) As shown in Table 4, the costs of the cirrhotic compensatory period and decompensated period of cirrhosis mainly included the direct medical cost of the patient, including outpatient fees, hospitalization fees, and medicine fees; direct non-medical costs included travel expenses, care worker fees, nutrition fees, etc. Liver cancer status costs mainly included direct hospitalization costs including medical expenses, nutritional expenses, travel expenses, nursing workers, etc. Outpatient clinic direct expenses included medical expenses, nutritional expenses, and travel expenses.

Table 4

Cost parameters and data sources in Markov model

\begin{tabular}{|c|c|c|c|c|}
\hline \multicolumn{2}{|l|}{ Project } & \multirow{2}{*}{$\begin{array}{l}\text { Value } \\
5775.1\end{array}$} & \multirow{2}{*}{$\begin{array}{l}\text { SD } \\
2669.71\end{array}$} & \multirow{2}{*}{$\begin{array}{l}\text { Distributed } \\
\text { Gamma }\end{array}$} \\
\hline Cost / year (yuan) & $\begin{array}{l}\text { Average annual cost of traditional } \\
\text { Chinese medicine }\end{array}$ & & & \\
\hline & $\begin{array}{l}\text { Average annual cost of antiviral } \\
\text { drugs }\end{array}$ & 2234.4 & 1586.22 & Gamma \\
\hline & $\begin{array}{l}\text { Average annual cost of liver } \\
\text { cancer }\end{array}$ & 62453.71 & 58642.54 & Gamma \\
\hline \multirow{2}{*}{$\begin{array}{l}\text { Hospitalization } \\
\text { expenses / time }\end{array}$} & Compensated cirrhosis ${ }^{[3]}$ & 20734.87 & 15969.03 & Gamma \\
\hline & Decompensated cirrhosis ${ }^{[3]}$ & 25328.69 & 20249.7 & Gamma \\
\hline \multirow{2}{*}{$\begin{array}{l}\text { Number of } \\
\text { hospitalizations / year }\end{array}$} & Compensated cirrhosis [3],[12-13] & 1.53 & 0.267 & Normal \\
\hline & Decompensated cirrhosis ${ }^{[3],[12-13]}$ & 1.81 & 0.317 & Normal \\
\hline \multicolumn{5}{|c|}{$\begin{array}{l}\text { Note: annual direct hospitalization cost = single hospitalization cost } x \text { number of hospitalizations; The } \\
\text { annual direct hospitalization cost of traditional Chinese medicine combined with antiviral drugs = } \\
\text { single hospitalization cost x number of hospitalizations x hospitalization impact coefficient; Total } \\
\text { cost = annual direct hospitalization cost + drug expense. The drug expense data was derived from } \\
\text { website the China } 315 \text { drug price, and the cost was discounted to the } 2018 \text { price level according to the } \\
\text { standards of the "Chinese Pharmaceutical Economics Evaluation Guide". }\end{array}$} \\
\hline
\end{tabular}

Model Construction and Operation 
The Markov model of the transition of various disease states of hepatitis B cirrhosis was shown in Fig. 1.

The parameters were brought into Markov model and simulated by TreeAge software to calculate the health output and economic cost of Chinese medicine combined with antiviral drugs and antiviral drugs alone in the treatment of hepatitis B cirrhosis 20 years. Markov model simulation operation process was shown in Fig. 2.

\section{Results}

\section{Health Output Analysis}

In the Markov model simulation of 20-years treatment, the probability distribution of each disease state in each cycle of hepatitis B cirrhosis treated by traditional Chinese medicine combined with antiviral drugs and antiviral drugs alone was shown in Fig. 3 and 4.

\section{Fig. 3 The probability distribution of each state in each cycle of NAs group in the Markov model}

\section{Fig. 4 Probability distribution of each state in each cycle of NAs + TCM group in Markov model}

Note: NAs: treatment with antiviral drugs alone; NAs + TCM: combination of traditional Chinese medicine and antiviral drugs.

As shown in Fig. 5, the probability curve of compensated cirrhosis of Chinese medicine combined with antiviral drugs in the treatment of hepatitis B cirrhosis was higher than that of antiviral drugs alone, indicating that Chinese medicine combined with antiviral drugs could prolong the disease progression of patients with compensated cirrhosis. The quality of life of patients with compensated cirrhosis was significantly higher than that of patients with decompensated cirrhosis and liver cancer.

\section{Fig. 5 Probability distribution of compensated cirrhosis of the two treatment options in each cycle}

As shown in Fig. 6, the probability curves of traditional Chinese medicine combined with antiviral drugs in the treatment of hepatitis B cirrhosis decompensated cirrhosis and antiviral drugs alone increased first and then decreased. The rate of increase and decrease of the probability curve of antiviral drugs alone was higher than that of traditional Chinese medicine combined with antiviral drugs. The antiviral drugs used alone reached the highest value of $19.32 \%$ in the sixth cycle, and the combined antiviral drugs of traditional Chinese medicine reached the highest value of $16.22 \%$ in the tenth cycle. In the first 11 cycles, the probability of using antiviral drugs alone in decompensated liver cirrhosis was higher than that of traditional Chinese medicine combined with antiviral drugs. The use of antiviral drugs alone after 11 cycles had a higher probability of decompensated liver cirrhosis than antiviral drugs alone.

Fig. 6 Probability distribution of decompensated cirrhosis of the two treatment options in each cycle 
As shown in Fig. 7, the probability curve of liver cancer treated with traditional Chinese medicine combined with antiviral drugs for hepatitis B cirrhosis was slightly higher than that of antiviral drugs alone.

\section{Fig. 7 Probability distribution of liver cancer status of two treatment options in each cycle}

As shown in Fig. 8, the mortality probability curve of traditional Chinese medicine combined with antiviral drugs for hepatitis $B$ cirrhosis was lower than that of antiviral drugs alone. The cumulative mortality rate after 20 years of treatment with antiviral drugs alone was $82.28 \%$, and the cumulative mortality rate of traditional Chinese medicine combined with antiviral drugs was $67.18 \%$. It suggested that the treatment of hepatitis B cirrhosis with Chinese medicine combined with antiviral drugs could reduce the mortality of hepatitis B cirrhosis and prolong the life cycle of patients.

Fig. 8 Probability distribution of death status of two treatment options in each cycle

\section{Cost Analysis}

The Markov model analysis result showed that the cumulative cost of the treatment plan of Chinese medicine combined with antiviral drugs was 337739.08 yuan, and the cumulative cost of the treatment plan of antiviral drugs alone was 383290.69 yuan. The total cost of traditional Chinese medicine combined with antiviral drugs for the treatment of hepatitis B cirrhosis was lower, and the total cost savings were 45551.61 yuan. The total cost savings were mainly due to the reduction in the number of hospitalizations caused by serious complications treated with traditional Chinese medicine combined with antivirals.

\section{Cost-Utility Analysis}

As shown in Table 5, the result of the model analysis showed that the cost of traditional Chinese medicine combined with antiviral treatment was 337739.08 yuan, and 6.22 QALYs has been obtained, with a cost-utility ratio of 54331.18 yuan / QALY. The cost of using antiviral drugs alone was 383290.69 yuan, and 4.74 QALYs were obtained, with a cost- utility ratio of 80844.37 yuan / QALY. The use of antiviral drugs alone was more costly and had a lower utility value than treated by traditional Chinese medicine combined with antiviral drugs.

Table 5 Cost-effectiveness analysis of traditional Chinese medicine combined with antiviral drugs and antiviral treatment alone for hepatitis B cirrhosis

\begin{tabular}{lllllll} 
Treatment & $\mathrm{U}(\mathrm{Q} A L Y S)$ & $\mathrm{C}$ & $\mathrm{U} / \mathrm{C}$ & $\triangle \mathrm{C}$ & $\triangle \mathrm{U}$ & $\triangle \mathrm{C} / \triangle \mathrm{U}(\mathrm{ICUR})$ \\
\hline TCM+NAs & 6.22 & 337739.08 & 54331.18 & -- & -- & -- \\
\hline NAs & 4.27 & 383290.69 & 80844.37 & 45551.61 & -1.48 & -30878.06
\end{tabular}




\section{Sensitivity Analysis}

Univariate sensitivity analysis and probabilistic sensitivity analysis were used to test the stability of the result. In univariate sensitivity analysis, the parameters of cost, utility value, transition probability and other parameters in the model were adjusted by $10 \%$. Adjusted the parameter (rr_t) of the influence of the traditional Chinese medicine combined with antiviral treatment on the number of hospitalizations from 0.5 to 1 . Adjusted the discount rate (rate) from $0 \%$ to $10 \%$. Analyze the influence of each parameter on the result and present it in the form of a cyclone chart. Probability sensitivity analysis adopted Monte Carlo sampling method to simulate 1000 random samples, and used cost-utility scatter plot to show the result.

\section{Single Factor Sensitivity Analysis}

The result of the cyclone graph showed that the model parameters that have a greater impact on the result were factors such as the effect of Chinese medicine combined with antiviral treatment on the number of hospitalizations ( $\left(r \_t\right)$, discount rate (rate), and the number of compensatory cirrhosis hospitalizations (t_cc). The effect of Chinese medicine combined with antiviral treatment on the number of hospitalizations ( $\mathrm{rr}_{-} \mathrm{t}$ ) had the greatest impact on the result (see Fig. 9). Univariate sensitivity analysis showed that, except for the effect parameter $\left(r r_{-} t\right)$ of Chinese medicine combined with antiviral therapy on the number of hospitalizations, the changes of other factors did not change the model result.

\section{Fig. 9 Cyclone diagram of single factor sensitivity analysis}

\section{Analysis of the impact of Chinese medicine combined with antiviral treatment on the number of hospitalizations}

Research showed that the direct cost of hospitalization accounted for $86.5 \%$ of the annual direct cost of hepatitis B cirrhosis [3]. Complications of hepatitis B cirrhosis were the leading cause of hospitalization for patients with cirrhosis [19]. Therefore, the parameter (rr_t) of the number of hospitalizations was selected to conduct a univariate sensitivity analysis on the result of Chinese medicine combined with antiviral drugs in the treatment of hepatitis B cirrhosis, and the result were shown as follows:

When the impact of the number of hospitalizations (rr_t) varied between 0.5 and 0.625 , the cost of using antiviral treatment alone was 383290.69 yuan, and 4.74 QALYs were obtained. The cost of using Chinese medicine combined with antiviral drugs varied between $315959.34 \sim 361333.80$ yuan, and 6.22 QALYs were obtained. The use of antiviral drugs alone was more costly and had a lower utility value than using traditional Chinese medicine combined with antiviral drugs. When the impact of the number of hospitalizations (rr_t) changed between $0.75 \sim 1$, the cost of using antiviral drugs alone was 383290.69 yuan, and 4.74 QALYs were obtained; The cost of using Chinese medicine combined with antiviral treatment varied between $406708.25 ~ 497457.16$ yuan, and 6.22 QALYs were obtained; the combination of Chinese medicine and antiviral drugs had the advantage of healthy output. The incremental cost of 
Chinese medicine combined with antiviral drugs varied from 23417.55 to 114166.46 yuan, the incremental utility was 1.48 QALYs, and the incremental cost-effectiveness ratio varied from 15874.05 to 77389.98 yuan / QALY.

As shown in Table 6, when the impact of the number of hospitalizations was adjusted to the maximum value ( $\left.r r_{-} t=1\right)$, that was, the annual hospitalization times of traditional Chinese medicine combined with antiviral drugs were the same as those of antiviral drugs alone, the cost of using antiviral drugs alone was 383290.69 yuan, and 4.74 QALYs were obtained; The cost of using Chinese medicine combined with antiviral drugs was 497457.16 yuan, and 6.22 QALYs were obtained. The combination of Chinese medicine and antiviral drugs had the advantage of healthy output. The incremental cost of Chinese medicine combined with antiviral treatment were 114166.46 yuan, the incremental utility was 1.48 QALYs, and the incremental cost- utility ratio was 77389.98 yuan / QALY.

According to the recommendations of the World Health Organization Macroeconomics and Health Committee (2011) [5]: when ICUR <1 times GDP per capita meant that the treatment plan was completely worthwhile; when ICUR <3 times GDP per capita meant that the treatment plan was acceptable; when ICUR> 3 times GDP per capita meant that the treatment plan was not worth investing. In 2018, China 's per capita GDP was 64644.0 yuan [20]. Sensitivity analysis result showed that when the parameter of the number of hospitalizations was taken the maximum value 1 ( $r$ _t $=1$ ), the incremental cost-utility ratio of traditional Chinese medicine combined with antiviral treatment was 77389.98 yuan / QALY, 1 times GDP per capita <ICUR <3 times GDP per capita. Therefore, the cost-effectiveness of traditional Chinese medicine combined with antiviral treatment was still acceptable.

Table 6 When the annual hospitalization time of traditional Chinese medicine combined with antiviral drugs is the same as that of antiviral drugs alone $\left(r r_{-} t=1\right)$

\begin{tabular}{llllll} 
Treatment & $\mathrm{C}$ & $\mathrm{U}(\mathrm{QALYs})$ & $\triangle \mathrm{C}$ & $\triangle \mathrm{U}$ & $\triangle \mathrm{C} / \triangle \mathrm{U}$ ICUR囚 \\
\hline NAs & 383290.69 & 4.74 & -- & -- & -- \\
\hline TCM+NAs & 497457.16 & 6.22 & 114166.46 & 1.48 & 77389.98
\end{tabular}

\section{Probability Sensitivity Analysis}

As shown in Fig. 10, the Monte Carlo sampling method was used to simulate 1000 random sampling times, and the cost-utility scatter plot of 1000 simulation times was obtained.

\section{Fig. 10 Monte Carlo 1000-time scatter plot}

As shown in Fig. 11, when the willingness-to-pay value was 8227.42 yuan, the most cost-effective probability of Chinese medicine combined with antiviral drugs and antiviral drugs alone for hepatitis B 
cirrhosis was $50 \%$; with the increase of willingness-to-pay value, the probability of cost-effectiveness of traditional Chinese medicine combined with antiviral therapy gradually increased, and the probability of cost-effectiveness of antiviral therapy alone decreased gradually; When the willingness-to-pay value was double GDP per capita (64644.0 yuan), the probability of cost-effectiveness of Chinese medicine combined with antiviral therapy was $88.3 \%$, and the probability of cost-effectiveness of antiviral therapy alone was $11.7 \%$.

\section{Fig. 11 Willingness-to-pay curve}

As shown in Fig. 12, the Monte Carlo sampling method was used to simulate 1000 random sampling times, and 1000 incremental cost-utility scatter points were obtained, of which $94.2 \%$ were distributed in quadrant I and quadrant IV. It showed that $94.2 \%$ probability that the incremental effect of using Chinese medicine combined with antiviral drugs was greater than $0 ; 86.7 \%$ was lower than 1 times GDP per capita. It showed that $86.7 \%$ of the simulation result were more cost-effective for the combination of traditional Chinese medicine and antiviral drugs.

\section{Fig. 12 Incremental cost-utility scatter plot}

\section{Discussion}

As traditional characteristic therapy in China, traditional Chinese medicine treatment could achieve the purpose of disease treatment by improving the patients' physique and enhancing the resistance to disease. It had a unique effect in chronic hepatitis B and other complex diseases [24]. At present, most of the treatment schemes of the combination of traditional Chinese and Western medicine were to make up for the shortcomings of the combination of drugs or to improve its efficacy. It was still necessary to improve and optimize the efficacy evaluation of large samples, strengthened the cooperative research with foreign well-known laboratories, and published high-quality academic papers.

At present, the incidence of hepatitis B in China was increasing year by year, and the age of onset was becoming younger, so effective preventive measures were particularly important [25]. Hepatitis $B$ vaccine could effectively block the spread of hepatitis $B$ virus, reduce the carrying and infection of hepatitis $B$ virus, so strengthen the vaccination related to hepatitis $B$ vaccine was necessary. However, hepatitis $B$ vaccine did not enter the medical insurance catalogue of our country, and the cost of vaccination was high, which might lead to the lower level of the overall vaccination of hepatitis B vaccine in our country, and the overall effect of chronic hepatitis B disease control was not very well.

As a chronic progressive liver disease, cirrhosis was a serious symptom after hepatitis. Because of the long treatment process, the treatment effect was difficult to see in a short time. The physical and psychological distress brought to patients were huge, and the quality of life of patients was generally low [23]. HBV could cause abnormal glucose metabolism, and gradually progress to diabetes, further 
aggravating the disease. With the improvement of living standard and social medical level, the increase of population aging and obesity and other problems, the patients with hepatitis B cirrhosis accompanied by diabetes, hypertension, dyslipidemia and obesity and other complications were increasing year by year. The demand for clinical treatment of hepatitis B cirrhosis was increasing year by year, and the government departments needed to pay enough attention.

Our research also had some limitations: 1 . There was no large-scale cohort study on Chinese medicine treatment of hepatitis B cirrhosis in China, the parameters in the model came from different research result, which might cause some confounding bias in the research result. 2. There was a lack of research on the effect of different treatment schemes on the number of hospitalizations of patients with hepatitis B cirrhosis. This factor has a greater impact on the result of the model, which needed to study in the future. 3. The calculation of the cost of antiviral drugs included the recommended drugs in the "Guidelines for the Prevention and Treatment of Chronic Hepatitis B" [4], such as entecavir, lamivudine and adefovir dipivoxil, This study did not consider drugs that were not in the guidelines, and also We did not consider factors related to changes in drug prices. Among them, entecavir was not within the patent protection period, and had entered the scope of medical insurance reimbursement in some regions of China, and the drug cost was relatively lower. However, the cost of drugs such as lamivudine and adefovir dipivoxil within the scope of patent protection was relatively higher, which may cause high calculation cost of antiviral drugs, and may have a certain impact on the result of the model. and the cost of drugs such as lamivudine and adefovir dipivoxil was relatively higher. Besides, the cost calculation of traditional Chinese medicines included prescriptions such as Fufang Biejia Ruangan Tablets, Ganshuang Granules, Jianganle Granules, Fuzheng Huayu Tablets, Anluo Huaxian Pills. The cost of traditional Chinese medicine treatment such as traditional Chinese medicine decoction was not included, therefore, there were some errors in the cost calculation of traditional Chinese medicine. This study performed a series of sensitivity analyses on drug prices and found that the result remain valid across a wide range of drug price. 4. The model did not include indirect costs in the simulation of long-term treatment, such as indirect costs related to productivity loss, which might have an impact on the model result. 5 . In this study, the discount rate of the model was determined according to "China Guidelines for Pharmacoeconomic Evaluations", rather than the GDP growth in China. The sensitivity analysis results of the discount rate showed that the change of the discount rate did not change the result.

\section{Conclusions}

The Markov model was used to simulate and evaluate the long-term treatment process of Chinese medicine combined with antiviral drugs in the treatment of hepatitis B cirrhosis. As described in this article, the result showed that compared with using antiviral drugs alone, the treatment of traditional Chinese medicine combined with antiviral drugs increased 1.48 QALYs per patient, and the improvement may be due to the lower cumulative incidence of related complications and the improvement of patients' quality of life. The total cost of treatment saved 45551.61 yuan, and the total cost savings mainly resulted from the reduction of hospitalization times caused by severe complications treated by the 
combination of traditional Chinese medicine and antiviral drugs. Therefore, the traditional Chinese medicine combined with antiviral treatment had cost-effectiveness advantages.

\section{Abbreviations}

HBV

hepatitis $B$ virus

WHO

World Health Organization

RR

relative risk

MD

mean difference

ICUR

incremental cost-utility ratios

QALY

quality-adjusted life year

CC

decompensated cirrhosis period

DC

decompensated cirrhosis period

HCC

liver cancer

Die

death

NAs

treatment with antiviral drugs alone

$\mathrm{NAs}+\mathrm{TCM}$

combination of traditional Chinese medicine and antiviral drugs.

SD

Standard deviation

\section{rr_t}

the effect of Chinese medicine combined with antiviral treatment on the number of hospitalizations rate

discount rate

t_cc

the number of compensatory cirrhosis hospitalizations

\section{Declarations}




\section{Ethics approval and consent to participate}

Not applicable.

\section{Consent for publication}

Not applicable.

\section{Availability of data and material}

Not applicable.

\section{Competing interests}

Not applicable.

\section{Funding}

No funding for this research.

\section{Authors' contributions}

Thank you very much, Professor Zhu Wentao, for your guidance.

\section{Acknowledgements}

Not applicable.

\section{References}

[1] WHO. Global hepatitis report,2017[EB/OL].[2019-11-06]. https://www. who.

int/hepatitis/publications/globalhepatitis-report2017/en/.

[2] D'AMICO G, GARCIA-TSAO G, PAGLIARO L. Natural history and prognostic indicators of survival in cirrhosis[J]. J Hepatol Int, 2006, 44(1):217-231. DOI: 10.1016/j.jhep.2005.10.013

[3] Ma Qishan, Liang Sen, Xiao Hewei, et al. Survey of economic burden of hepatitis B-related diseases in 12 areas in China. Chinese journal of Epidemiology, 2017, 38(7): 868-876. DOIه10.3760/cma.j.issn.02546450.2017.07.005. 
[4] Wang Guiqiang, Duan Zhongping et al. The guidelines of prevention and treatment for chronic hepatitis B(2019 version) [J/OL]. Journal of Practical Hepatology:1-22[2019-12-24].

DOI:10.12037/YXQY.2019.12-09

[5] Liu Guoen, Hu Shanlian, Wu Jiuhong. China Guidelines for Pharmacoeconomic Evaluations (2011 version) [J]. China Journal of Pharmaceutical Economics,2011(03):6-9+11-48.

https://kns8.cnki.net/kns/detail/detail.aspx?FileName=ZYWA201103002\&DbName=CJFQ2011

[6] Giovanna Fattovich, Flavia Bortolotti, Francesco Donato. Natural history of chronic hepatitis B: Special emphasis on disease progression and prognostic factors[J]. Journal of Hepatology,2007,48(2). DOI:10.1016/j.jhep.2007.11.011

[7] Xu Biao, Jiang Qingwu, DM Rosenberg, et al. The relationship between seroconversion of ant-HBe and disease progression in patients with chronic hepatitis B. Journal of Clinical Hepatology,2003,19(1):16-18. https://kns8.cnki.net/kns/detail/detail.aspx?FileName=LCGD200301007\&DbName=CJFQ2003

[8] Wong JB, Koff RS, Tine F, Pauker SG. Cost effectiveness of interferon-alpha 2b treatment for hepatitis B e antigen-positive chronic hepatitis B. Ann Intern Med 1995,122:664-775. DOI: 10.7326/0003-4819-1229-199505010-00004

[9] Chen Dong, Yao Guangbi, Chen Wen. Economic evaluation of peginterferon Alfa-2a and lamivudine in the treatment of HBeAg-negative chronic hepatitis B. [J]. Chinese Hepatology,2007(03):164-167. DOI区 10.14000/j.cnki.issn.1008-1704.2007.03.005

[10] David L. Veenstra, Sean D. Sullivan, Ming-Yang Lai, et al. HBeAg-Negative Chronic Hepatitis B: CostEffectiveness of Peginterferon Alfa-2a Compared to Lamivudine in Taiwan[J]. Value in Health,2007,11(2). DOI:10.1111/j.1524-4733.2007.00221.x

[11] Feng Shuo. Methodological Study on Systematic Evaluation and Bias of Conclusions in Treatment of Chronic Hepatitis B Virus Carrier, Liver Fibrosis and Cirrhosis[D]. Beijing University of Chinese Medicine, 2017. https://kns8.cnki.net/kns/detail/detail.aspx?FileName=1017183640.nh\&DbName=CDFD2017

[12] Zheng Xiang. Analysis of the economic burden and influencing factors of hepatitis B related diseases in Taizhou city of Zhejiang Province[D]. Zhejiang University,2018.

https://kns8.cnki.net/kns/detail/detail.aspx?FileName=1018221260.nh\&DbName=CMFD2018

[13] Zhang Hua. Study on Disease Burden and Control Evaluation of Hepatitis B in Jiangsu Province[D]. Southeast University,2015. https://kns8.cnki.net/kns/detail/detail.aspx?

FileName $=1016215490$. nh\&DbName $=$ CDFD2017

[14] Fattovich G, Bortolotti F, Donato F. Natural history of chronic hepatitis B: special emphasis on disease progression and prognostic factors[J]. J Hepatology,2008,48(2):335-352. DOI:10.1016/j.jhep.2013.02.015 
[15] Wang Fuzhen, Cui Fuqiang, Zheng Hui et al. Evaluation on Protective Efficacy of Recombinant Yeast Derived Hepatitis B Vaccines Combination With Hepatitis B Immune Globulin in Preventing Mother-toinfant Transmission of Hepatitis B Virus. Chinese Journal of Vaccines and Immunization,2007(04):293297. http://kns.cnki.net/kcms/detail/detail.aspx?FileName=ZGJM200704002\&DbName=CJFQ2007

[16] Guo Jiewan, Jiang Kaiping, Hu Hongtao, et al. Discussion on the Treatment of Hepatitis B Cirrhosis with Tonifying the Kidney and Filling the Essence[J]. Journal of Liaoning University of Traditional Chinese Medicine,2019,21(12):190-193. DOI:10.13194/j.issn.1673-842x.2019.12.050

[17] Liaw Y F, Chu C M, Hepatitis B virus infection[J]. Lancet, 2009,373(9663):582-592.

DOI:10.1016/S0140-6736(09)60207-5

[18] Mcmahon B J. Natural history of chronic hepatitis B[J]. Clin Liver Dis,2010,14(3):381-396. DOI:10.1016/j.cld.2010.05.007.

[19] Jia Jidong, Wei Lai, Hou Donglin, et al. Chinese standards for diagnosis and treatment of liver disease-White paper excerpt[J]. Journal of Clinical Hepatology,2014,30(03):197-209.

DOI:10.3969/j.issn.1001-5256.2014.03.002

[20] Statistical Communiqué of the People's Republic of China on National Economic and Social Development in 2018 [J]. China Statistics,2019(03):8-22. http://kns.cnki.net/kns/detail/detail.aspx? FileName=RMRB201903010100\&DbName $=$ CCND2019

[21] Wang Dahan, Yang Chao. Evaluation of the application effect of stress and adaptation theory in the nursing of patients with compensated liver cirrhosis [J]. Chinese General Practice Nursing,2020,18(06):742-743+749. DOI:10.12104/j.issn.1674-4748.2020.06.021

[22] Shen Tian, Zhuang Li, Sun Xiaodong, et al. Management of clinical diagnosis, evaluation, and antiviral therapy for HBV-related cirrhosis [J]. Journal of Clinical Hepatology,2019,7(06):409-416. DOI:10.3969/j.issn.1674-7445.2020.01.004

[23] Lai Tao, Effect of extended care on quality of life in patients with post-hepatitis cirrhosis [J]. Electronic Journal of Practical Clinical Nursing Science,2018,3(04):26+30.

http://kns.cnki.net/kcms/detail/detail.aspx?FileName=SLHL201804022\&DbName=CJFQ2018

[24] Tang Yuehua, Ding Xianjun, Zhou Guoer, et ai. Clinical Study on Hepatic Fibrosis of Chronic Hepatitis B Treated with Jianpi Ruangan Granule [J]. Chinese Journal of Traditional Medical Science and Technology,2019,26(3):331-334. http://kns.cnki.net/kcms/detail/detail.aspx?

FileName=TJYY201903004\&DbName=CJFQ2019

[25] Chen Qiong. The application of nursing guidance in children's hepatitis B vaccination [J]. Journal of Electrocardiogram (Electronic Edition),2020,9(01):184-185. http://kns.cnki.net/kcms/detail/detail.aspx? FileName $=$ XDTZ202001151\&DbName=CJFQTEMP 
[26] Das Kausik, Das Kshaunish, Datta Simanti, et al. Course of disease and survival after onset of decompensation in hepatitis B virus-related cirrhosis [J]. Liver international: official journal of the International Association for the Study of the Liver,2010,30(7). DOI:10.1111/j.1478-3231.2010.02255.x

\section{Figures}

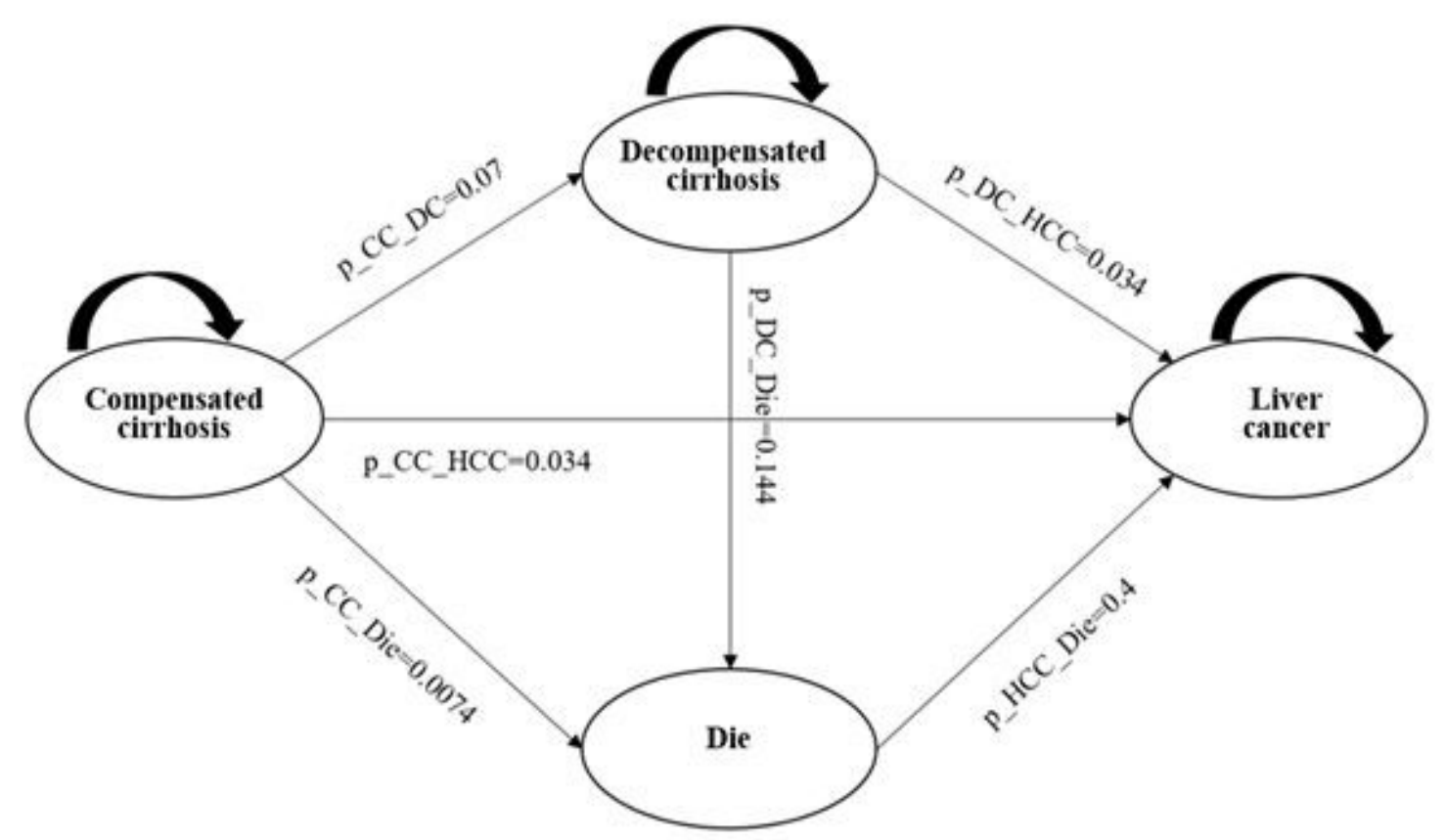

Figure 1

Markov model of various disease states of hepatitis B cirrhosis 


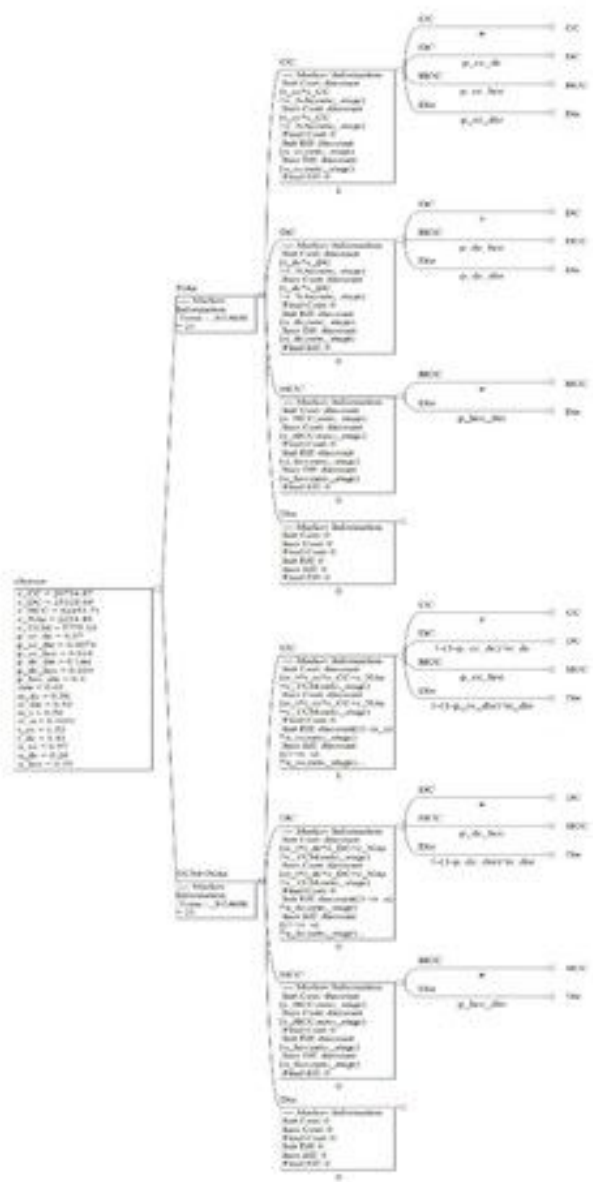

Figure 2

Markov model calculation process of two treatment schemes

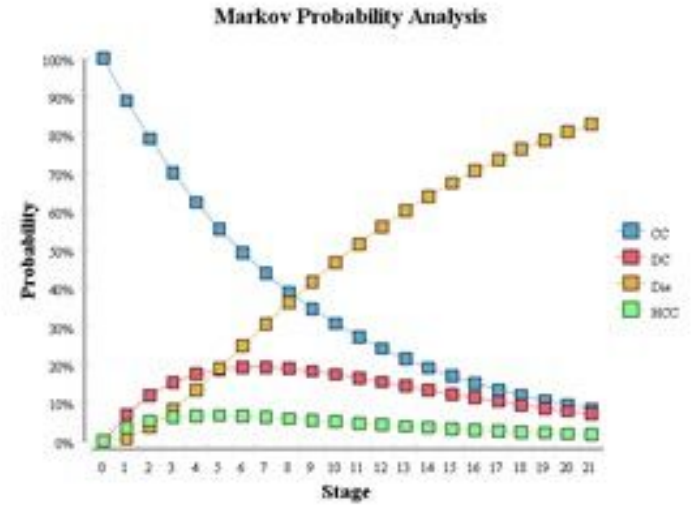

Figure 3

The probability distribution of each state in each cycle of NAs group in the Markov model 


\section{Markov Probability Analysis}

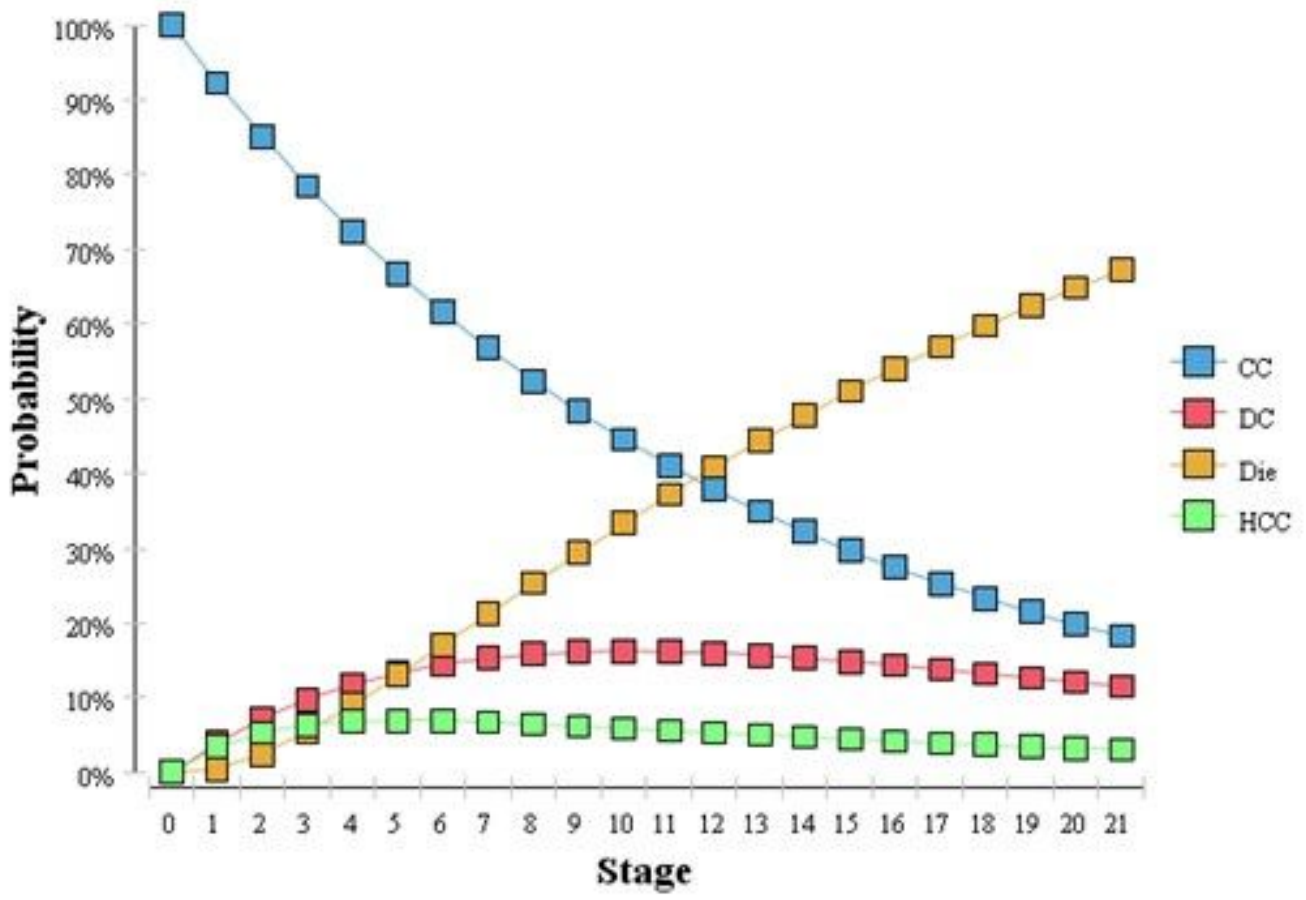

\section{Figure 4}

Probability distribution of each state in each cycle of NAs + TCM group in Markov model

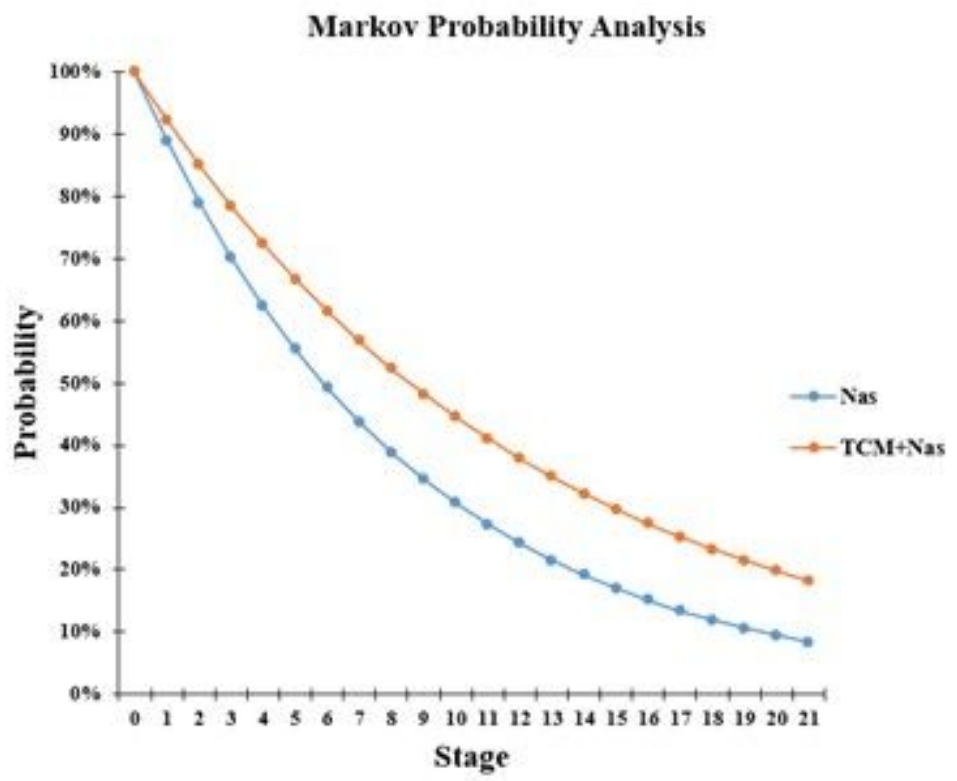

Figure 5

Probability distribution of compensated cirrhosis of the two treatment options in each cycle 


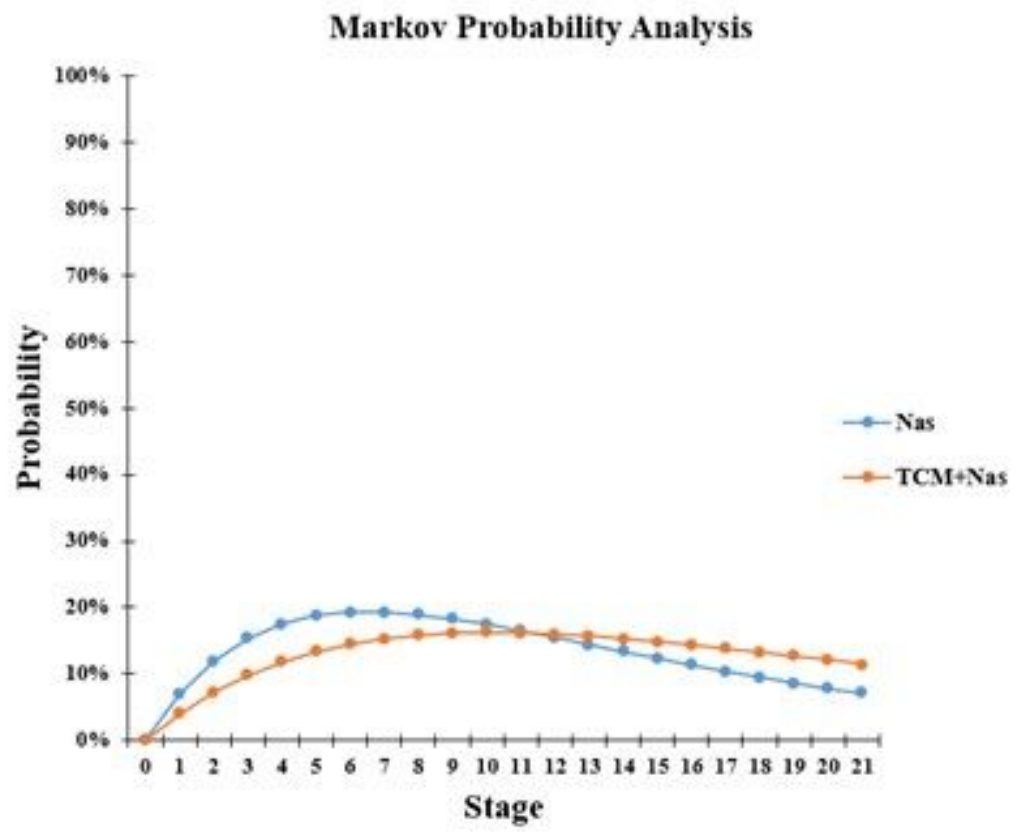

Figure 6

Probability distribution of decompensated cirrhosis of the two treatment options in each cycle

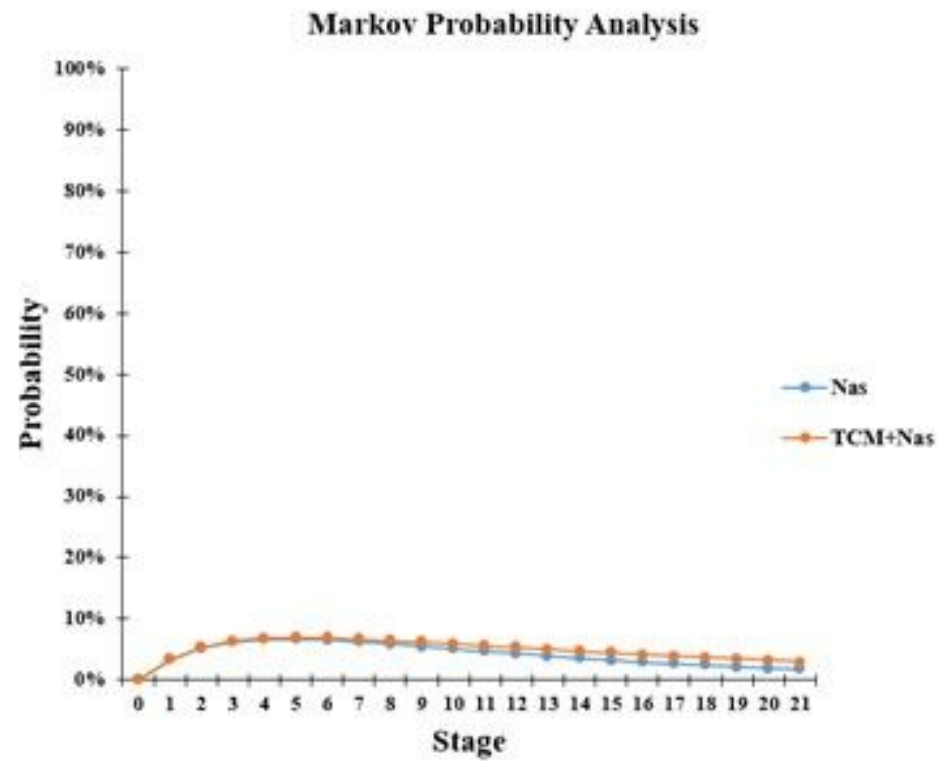

Figure 7

Probability distribution of liver cancer status of two treatment options in each cycle 


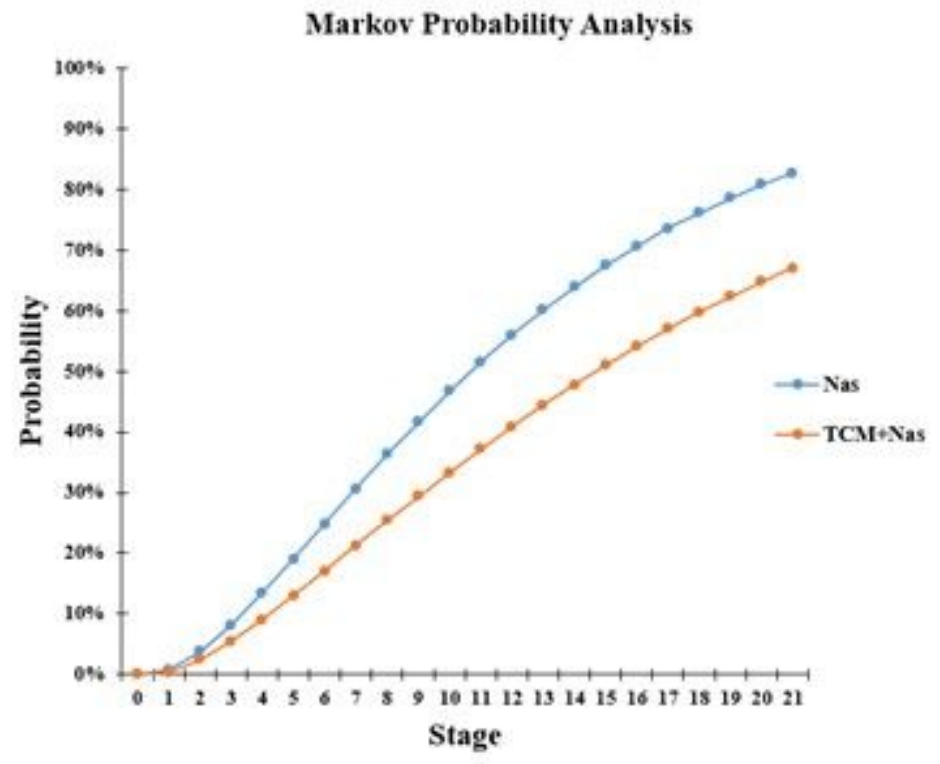

\section{Figure 8}

Probability distribution of death status of two treatment options in each cycle

\section{Tornado Analysis (Net Bene fits)}

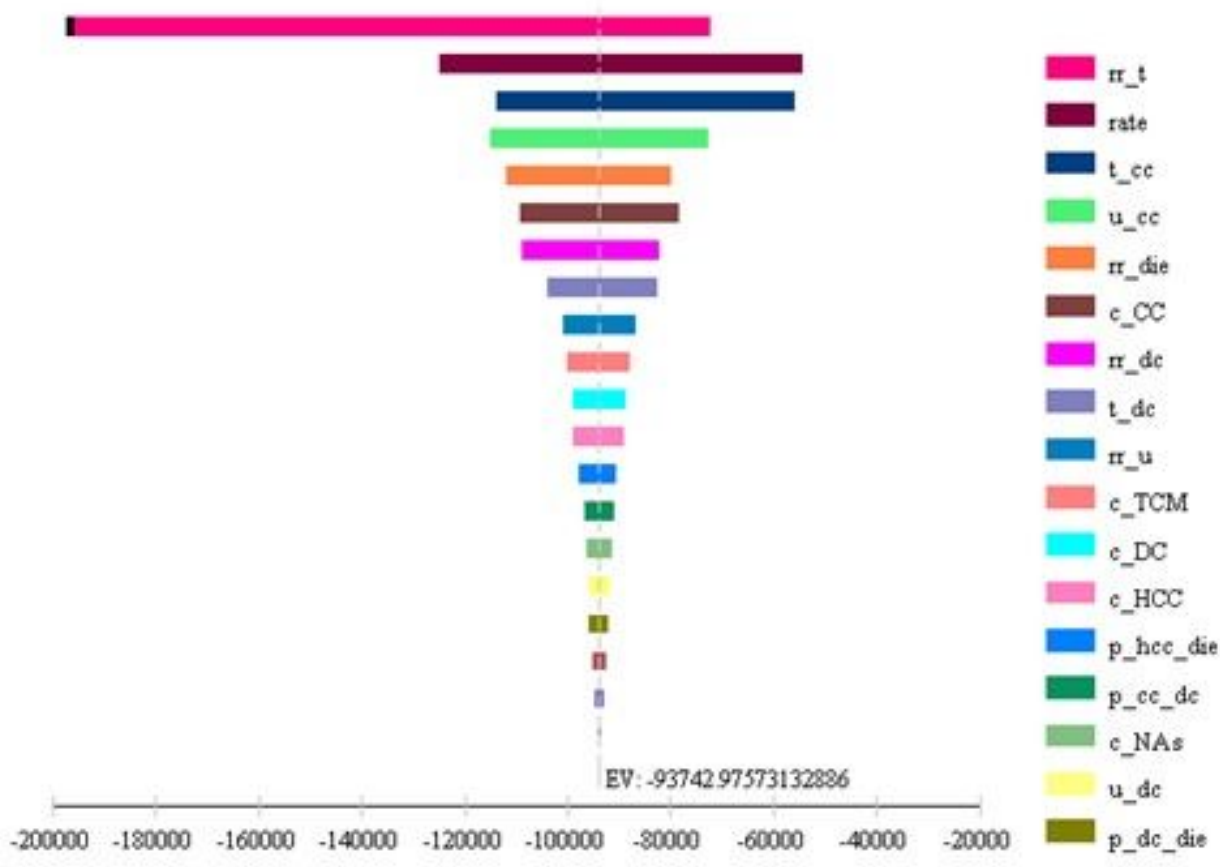

Fig. 9 Cyclone diagram of single factor sensitivity analysis

\section{Figure 9}


Cyclone diagram of single factor sensitivity analysis

\section{Cost-Effectiveness Scatterplot}

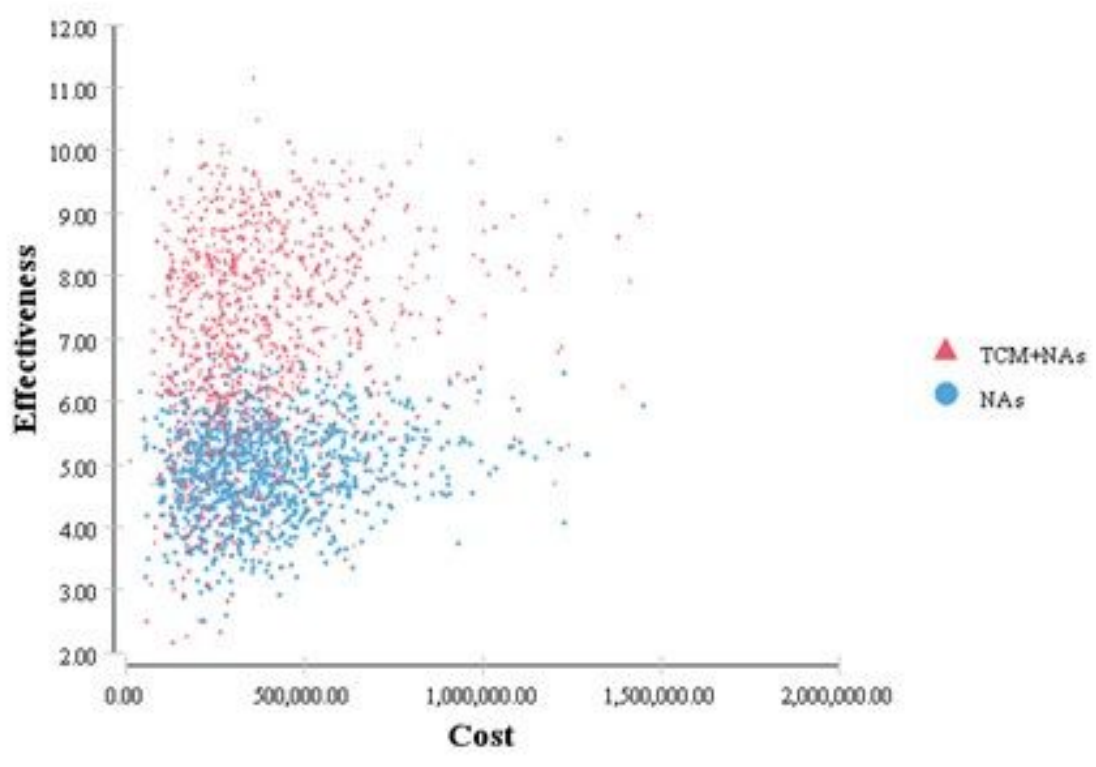

Fig. 10 Monte Carlo 1000-time scatter plot

Figure 10

Monte Carlo 1000-time scatter plot

\section{CE Acceptability Curve}

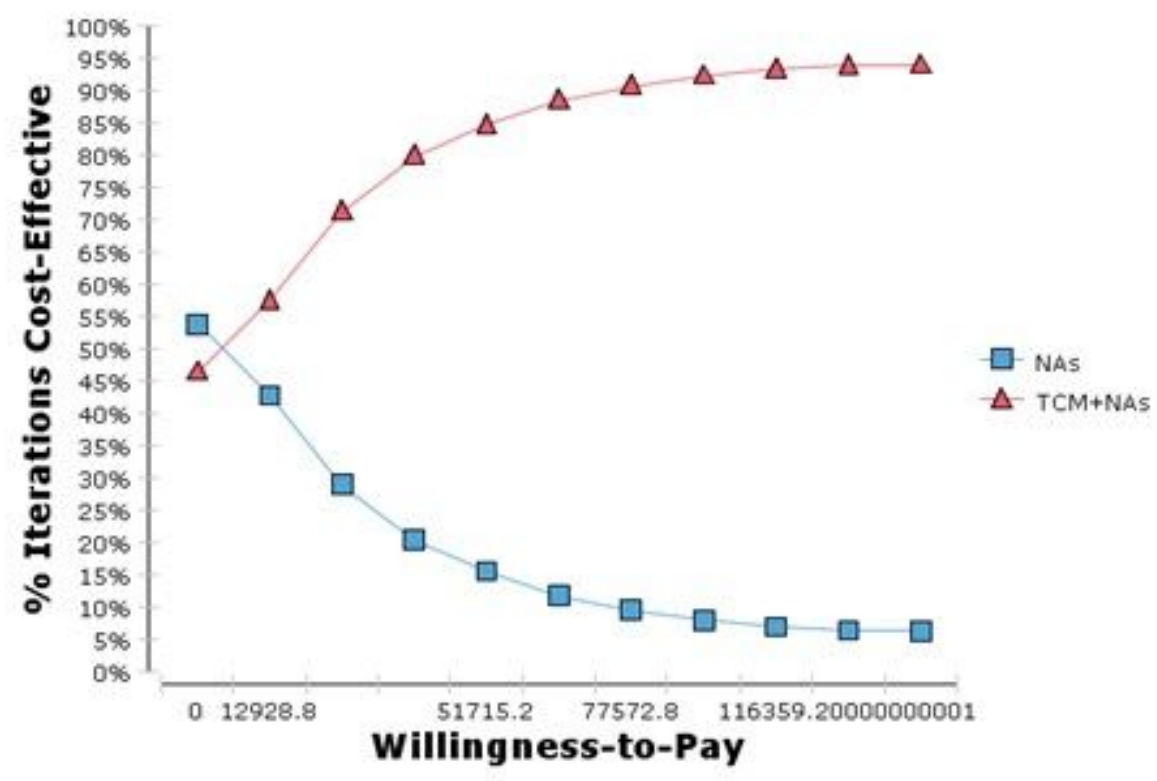

Fig. 11 Willingness-to-pay curve

\section{Figure 11}

Willingness-to-pay curve 
Incremental Cost-Effectiveness, TCM+NAs v. NAs

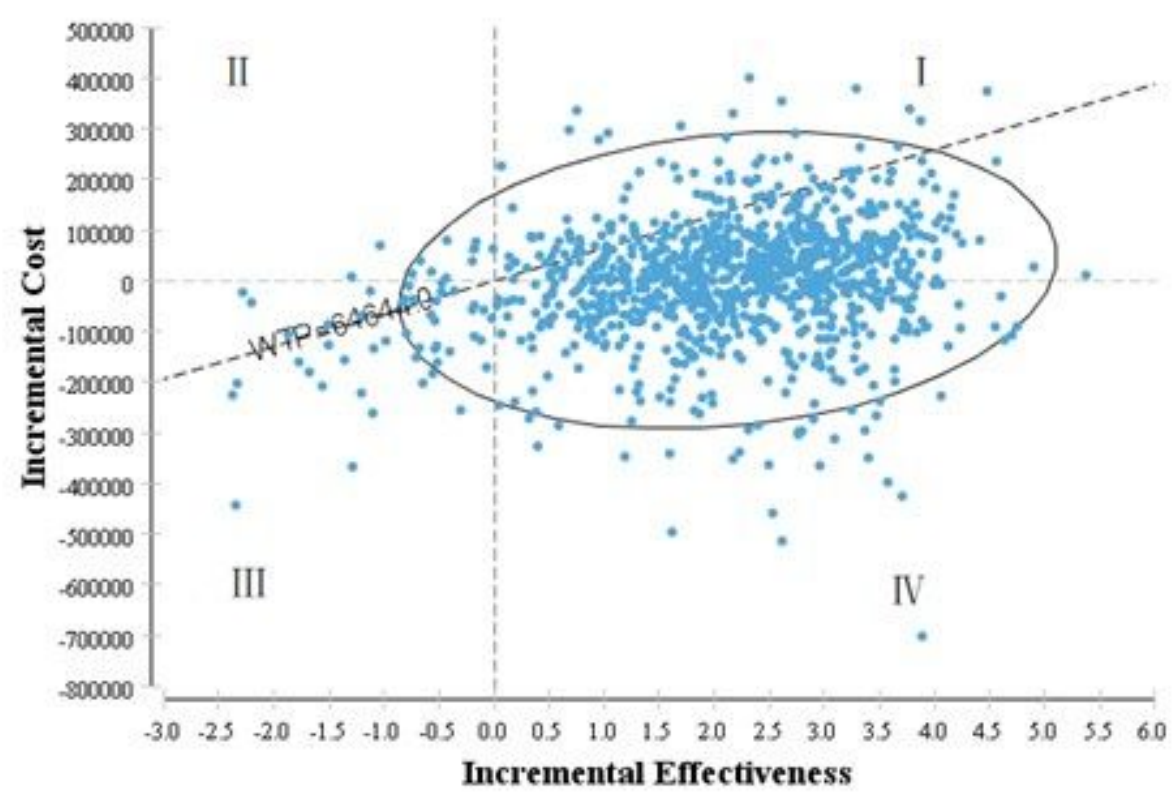

Figure 12

Incremental cost-utility scatter plot 